\title{
The Inflammatory and Hemostatic Response in Sepsis and Meningococcemia
}

\author{
Navin P. Boeddha, MD, PhD ${ }^{a}$, Thomas Bycroft, MD ${ }^{b}$, \\ Simon Nadel, MD ${ }^{b, c}$, Jan A. Hazelzet, MD, PhD ${ }^{d, *}$
}

\section{KEYWORDS}

- Bacterial infections • Bacteremia • Hemostasis • Host-pathogen interaction

- Inflammation • Meningococcal infections • Physiology • Sepsis

\section{KEY POINTS}

- Complex interplays among host, pathogen, and environment determine the severity of infection ranging from harmless nasopharyngeal colonization to bacteremia, meningitis, sepsis, and lethal disease.

- The inflammatory response includes proinflammatory and anti-inflammatory responses in innate and adaptive immunity. The net proinflammatory state causes endothelial dysfunction and activation of the hemostatic response.

- Within the wide range of illness severity, deposition of fibrin throughout the microcirculation could result in multiple organ dysfunction, skin grafts/amputation, and death.

- Meningococci hold unique properties to promote adhesion, colonization, and invasion into the bloodstream.

\section{INTRODUCTION}

Despite important reductions in the number of cases as a result of vaccination programs, Neisseria meningitidis (meningococcal) disease is still a major cause of invasive bacterial infections globally. ${ }^{1,2}$ Complex interplays among host, pathogen, and environment determine the severity of disease. ${ }^{3-6}$ The severity of infection ranges from harmless nasopharyngeal colonization to bacteremia, meningitis, sepsis, and lethal disease.

Meningococcemia refers to dissemination of meningococci into the bloodstream and is notorious for its rapid progression to fulminant disease. Meningococcal

\footnotetext{
a Department of Pediatrics, Erasmus MC-Sophia Children's Hospital, University Medical Center Rotterdam, Doctor Molewaterplein 40, 3015 GD Rotterdam, The Netherlands; ${ }^{b}$ St Mary's Hospital, Imperial College Healthcare NHS Trust, Praed Street, W21NY London, UK; ' Department of Paediatrics, Faculty of Medicine, Imperial College London, South Kensington Campus, London SW7 2AZ, UK; ${ }^{d}$ Department of Public Health, Erasmus MC, University Medical Center Rotterdam, Doctor Molewaterplein 40, 3015 GD Rotterdam, The Netherlands

* Corresponding author.

E-mail address: j.a.hazelzet@erasmusmc.nl
} 
endotoxins in the bloodstream and the subsequent inflammatory host response induce endothelial damage, excessive coagulation, and downregulation of fibrinolysis. Hence, the delicate balance between coagulation and anticoagulation shifts toward thrombosis and widespread deposition of fibrin throughout the microcirculation with thromboembolism contributing to multiple organ dysfunction and eventually death. ${ }^{7}$

Meningococcal sepsis serves as a unique model to study inflammation and coagulation in sepsis. Because of early recognition of clinical features, such as the characteristic rash and shock syndrome, pathophysiologic processes in the early phase of sepsis can be studied. In addition, meningococcal disease most commonly occurs in previously healthy children and the broad spectrum of illness severity enables the study of risk factors for adverse outcome. Lastly, meningococcal disease is still common, and thus relevant, in many countries across the world.

In this article, we review the pathogenesis of sepsis, in particular the inflammatory and hemostatic response in meningococcal sepsis. Reviews on meningococcal sepsis epidemiology, clinical features, management, and prevention are found elsewhere. .,9 $^{8}$

\section{INFLAMMATORY RESPONSE IN SEPSIS}

The inflammatory response ${ }^{10-12}$ to infection is characterized by two stages and includes innate and adaptive immune responses (Fig. 1). The first stage, a proinflammatory response, is initiated by pattern-recognition receptors of the innate immune system (eg, monocytes, macrophages, neutrophils, and dendritic cells) sensing pathogens (pathogen-associated molecular patterns) or stress signals (danger-associated molecular patterns). This detection leads to an intracellular signaling with activation of transcription factors, leading to the release of various proinflammatory cytokines (eg, tumor necrosis factor- $\alpha$, interleukin-1, interleukin-6) and chemokines that attract even more immune cells, enhancing phagocytosis. Additionally, proteins of the complement system (eg, C1q and mannan-binding lectin) bind to the surface of pathogens and augment their destruction.

These proinflammatory factors also mount a more specific adaptive immune response, which depends on antigen presentation via major histocompatibility complex molecules to lymphocytes. Two classes of lymphocytes, T cells and B cells, are responsible for cell-mediated immune responses and antibody responses, respectively. T cells directly recognize and destroy infected cells, whereas the production of antibodies against specific pathogens by B cells provides humoral immunity.

Simultaneous to the proinflammatory response, a systemic inhibition of the immune system occurs to restore homeostasis. ${ }^{13}$ The result is that monocytes and macrophages have diminished capacity to release proinflammatory cytokines on stimulation and blood monocytes are reprogrammed with reduced expression of HLA-DR. ${ }^{14,15}$ Additionally, there is an increase in T-cell apoptosis and release of antiinflammatory mediators to counteract continual inflammation. Thus, the innate and adaptive immune system contribute to sepsis-induced immunosuppression. ${ }^{10}$

Usually, the combined proinflammatory and anti-inflammatory response is able to combat the infection, without becoming unbalanced and harmful. However, an excessive proinflammatory response can result in early mortality in sepsis because of cardiovascular collapse and multiple organ dysfunction. In addition, an extended release of anti-inflammatory mediators (termed immunoparalysis) can potentially result in failure to clear primary infections and increases susceptibility to new infections, resulting in late sepsis mortality.

Meningococcemia is the result of meningococci evading the inflammatory host response. Pili are present on meningococci cell membrane and play a key role in 


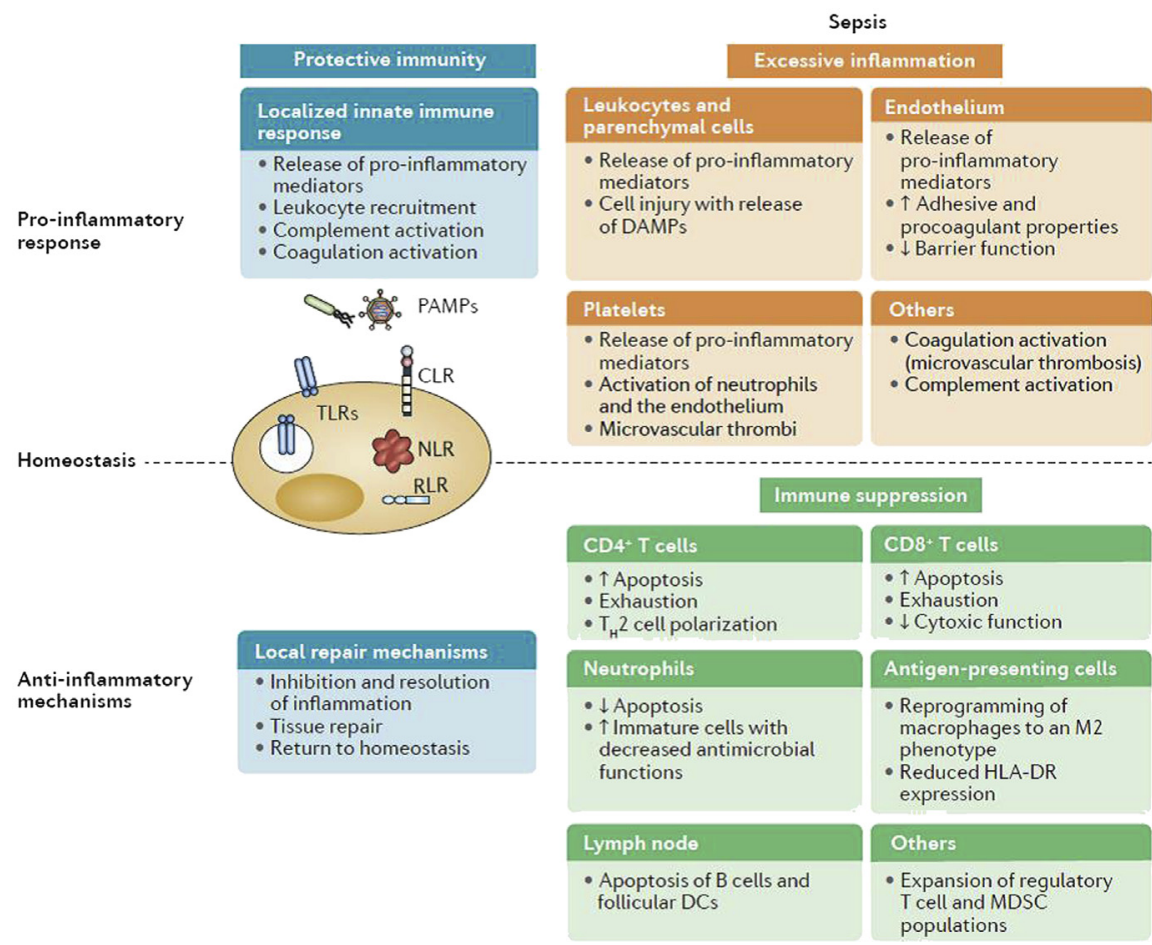

Fig. 1. The inflammatory response to sepsis. The inflammatory response includes a proinflammatory and an anti-inflammatory response. An initial proinflammatory response is initiated by PAMPs sensed by immune cells (eg, leukocytes and parenchymal cells, endothelial cells, and platelets) through an assortment of cell-surface and intracellular pattern recognition receptors (eg, TLRs, NLRs, RLRs, and CLRs). Various proinflammatory cytokines and chemokines are released to neutralize the infection. In addition, an anti-inflammatory compensatory mechanism restrains the initial inflammation, prevents collateral tissue damage, and restores homeostasis. An unbalanced and harmful response may result from prevailing and multiplying of the pathogen despite an activated immune response, leading to a concurrent excessive inflammation (top right). Extended release of anti-inflammatory mediators could result in immune suppression (bottom right). CLR, C-type lectin receptors; DAMPs, danger-associated molecular patterns; DCs, dendritic cells; MDSC, myeloid-derived suppressor cell; NLR, nucleotide-binding oligomerization domain-like receptors; PAMPs, pathogen-associated molecular patterns; RLR, retinoic acid-inducible gene-like receptors; TLRs, Toll-like receptors. (From van der Poll T, van de Veerdonk FL, Scicluna BP, et al. The immunopathology of sepsis and potential therapeutic targets. Nat Rev Immunol. 2017;17(7):407-20; with permission.)

adherence and nasopharyngeal colonization. Epithelial penetration is enhanced by phagocytic vacuoles and outer membrane proteins. ${ }^{16,17}$ After invasion into the bloodstream, the outer membrane is further used to evade the host immune response by inhibiting phagocytosis. ${ }^{16}$ Meningococci furthermore possess properties to inhibit host complement activation by binding of host complement factor $\mathrm{H}$ to the meningococcal factor $\mathrm{H}$-binding protein. ${ }^{18,19}$ Virulence factors of the meningococcal outer membrane, including outer membrane proteins and surface blebs containing lipopolysaccharide, function as endotoxin, and stimulation of various proinflammatory cytokines results in an excessive proinflammatory response. ${ }^{20}$ 


\section{HEMOSTATIC RESPONSE IN SEPSIS}

The hemostatic response $e^{21-23}$ is initiated because of endothelial activation and bystander damage after invasion of the bloodstream and inflammation activation by meningococci and endotoxins. Subsequently, tissue factor is released and increasingly expressed by endothelial cells (Fig. 2). The tissue factor-factor VII pathway ultimately results in the generation of thrombin, and the conversion of fibrinogen to fibrin.

Meningococci in the bloodstream adhere onto endothelial cells via pili, have the ability to resist high blood velocities, multiply, and form microcolonies on the apical surface of the endothelial cells. ${ }^{24}$ Subsequently, the integrity of the endothelium is challenged. Invasion of meningococci through the endothelium involves transcellular and paracellular processes: transcellular, via cell fenestrations or establishment of

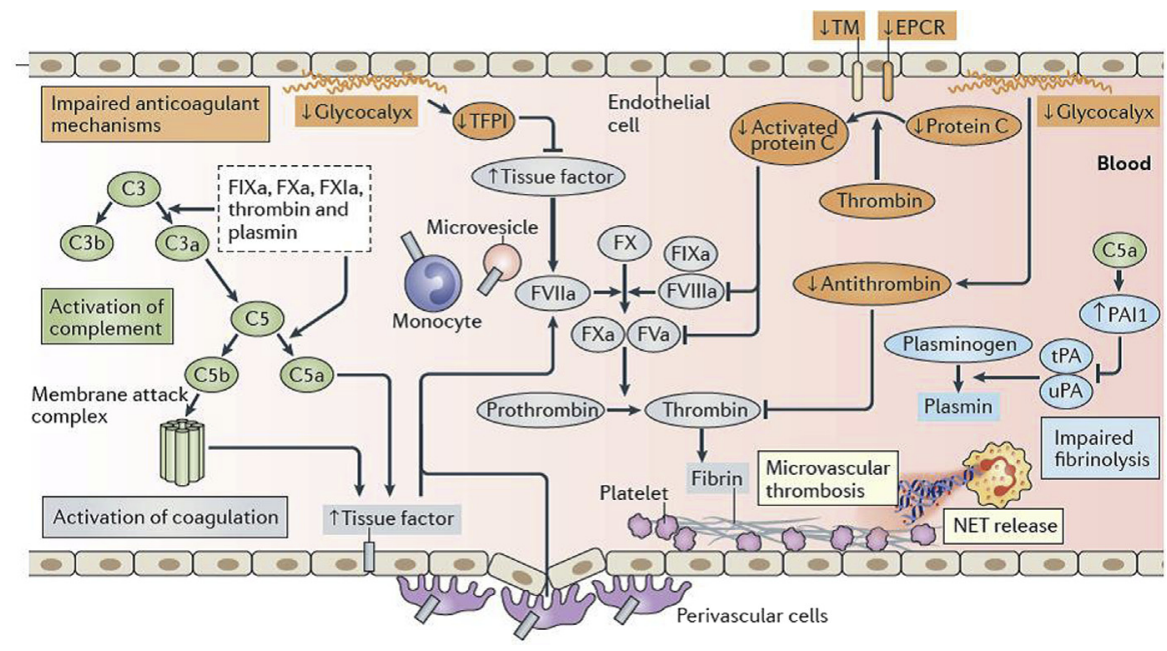

Fig. 2. The hemostatic response to sepsis. Sepsis results in a net procoagulant state in the microvasculature by at least three mechanisms. (1) Inflammatory cytokine-initiated activation of tissue factor generating thrombin (gray). Sepsis is accompanied by inflammationinduced vessel injury, which exposes tissue factor to blood coagulation factors, resulting in blood clotting. Tissue factor binds and activates FVII, after which a cascade of proteolytic reactions results in the formation of FXa, thrombin, and fibrin. (2) Insufficient control of anticoagulant pathways (orange). The tendency toward thrombosis during sepsis is augmented by the concurrently compromised activity of the three main anticoagulant pathways: antithrombin, TFPI, and the protein $C$ system. Antithrombin is the main inhibitor of thrombin and FXa, whereas TFPI is the main inhibitor of the tissue factor-FVIla complex. Activated protein $C$ is generated from protein $C$ at the surface of resting endothelial cells, a process that is mediated by the binding of thrombin to TM and is amplified by the EPCR. Activated protein C proteolytically inactivates the coagulation cofactors FVa and FVIIla, thereby inhibiting coagulation. During sepsis, the protein $C$ system is impaired as a result of multiple factors, most notably the decreased synthesis of protein $C$ by the liver, the increased consumption of protein $C$, and the impaired activation of protein $C$ as a result of diminished TM expression on endothelial cells. (3) PAI-1-mediated suppression of fibrinolysis (blue). The interaction with the complement system (green) is outside the scope of this review. EPCR, endothelial cell protein C receptor; NET, neutrophil extracellular traps; PAI, plasminogen activator inhibitor; TFPI, tissue factor pathway inhibitor; TM, thrombomodulin; tPA, tissue-plasminogen activator; uPA, urokinase plasminogen activator. (From van der Poll T, van de Veerdonk FL, Scicluna BP, et al. The immunopathology of sepsis and potential therapeutic targets. Nat Rev Immunol. 2017;17(7):407-20; with permission.) 
complex systems of vesiculovacuolar organelles, and paracellular based on the coordinated opening and closure of endothelial cell-cell junctions. ${ }^{25}$ These processes also account for the ability to cross the blood-brain barrier. ${ }^{26}$ Additionally, there is a direct interaction between bacteria and endothelial cells leading to a loss of integrity and increase in endothelial permeability. ${ }^{26}$ As a consequence, histology of skin lesions reveal bacteria within the endothelium and thrombi. ${ }^{27}$

In normal circumstances, activation of coagulation is controlled by three important physiologic anticoagulant pathways: (1) the antithrombin system, (2) tissue factor pathway inhibitor, and (3) the activated protein $C$ (PC) pathway. The main function of the PC pathway is to control coagulation by causing inactivation of activated (a) factor V (cofactor of factor Xa) and factor VIIla (cofactor of factor IX), subsequently preventing thrombin generation. ${ }^{28} \mathrm{PC}$ also neutralizes plasminogen activator inhibitor (PAI)-1. PAI-1, encoded by SERPINE1, is produced by endothelial cells. PAI-1 functions as the principal inhibitor of tissue plasminogen activator and urokinase plasminogen activator, and is the most important fibrinolytic inhibitor in vivo. Thus, PC concomitantly increases fibrinolytic capacity. Thrombomodulin, an endothelial cell surface glycoprotein, binds circulating thrombin and forms a thrombomodulinthrombin complex (Fig. 3). This complex rapidly activates PC bound to the endothelial cell $\mathrm{PC}$ receptor. Activated PC then dissociates from the endothelial cell PC receptor, binds to protein S, and forms a complex that inactivates factor Va and factor VIIla, thus reducing thrombin generation.

In sepsis, decreased activity of all three natural anticoagulant mechanisms results from a combination of impaired synthesis, ongoing consumption, leakage into the interstitial space, and proteolytic degradation. Decreased levels of $\mathrm{PC}^{29}$ and increased levels of PAI-1 ${ }^{30,31}$ are associated with a negative outcome in sepsis. In meningococcal disease, it has been shown clearly that $4 \mathrm{G} / 4 \mathrm{G}$ homozygotes have higher levels of PAI-1, which is associated with more severe disease. ${ }^{30}$

Altogether, these mechanisms in patients with sepsis result in coagulation abnormalities ranging from subtle derangements only detectable by highly sensitive assays to widespread deposition of fibrin throughout the microcirculation, manifesting as disseminated intravascular coagulation, as typically seen in meningococcal sepsis. Ultimately, disseminated intravascular coagulation contributes to multiple organ dysfunction and to the need for skin grafts, amputation of digits and extremities, and can eventually result in death from multiple organ dysfunction.

Genetic polymorphisms or combination of polymorphisms partly determine interindividual variety in the host response to infection and have been associated with susceptibility and severity of sepsis., ${ }^{4,32}$ A genome-wide association study, including approximately 1500 patients with meningococcal disease, reported an association between polymorphisms in the previously mentioned complement factor $\mathrm{H}$ region, which play a role in complement activation, and susceptibility. ${ }^{19}$ In adults with sepsis caused by pneumonia, a genome-wide association study revealed FER, which encodes a cytosolic nonreceptor tyrosine kinase that influences neutrophil chemotaxis and endothelial permeability, to be associated with a reduced risk of death. ${ }^{33}$ Although genetic variations have the potential to affect the host response to an infectious challenge, genetic findings have not been translated to clinical practice.

\section{Neutrophil Extracellular Traps as Cross-link Between Inflammation and Coagulation}

Neutrophils are an important part of the innate immune defense. They migrate to the site of infection to release regulatory cytokines, chemokines, and leukotrienes to contribute to microbial killing. ${ }^{34}$ One of the tools actively contributing to microbial 


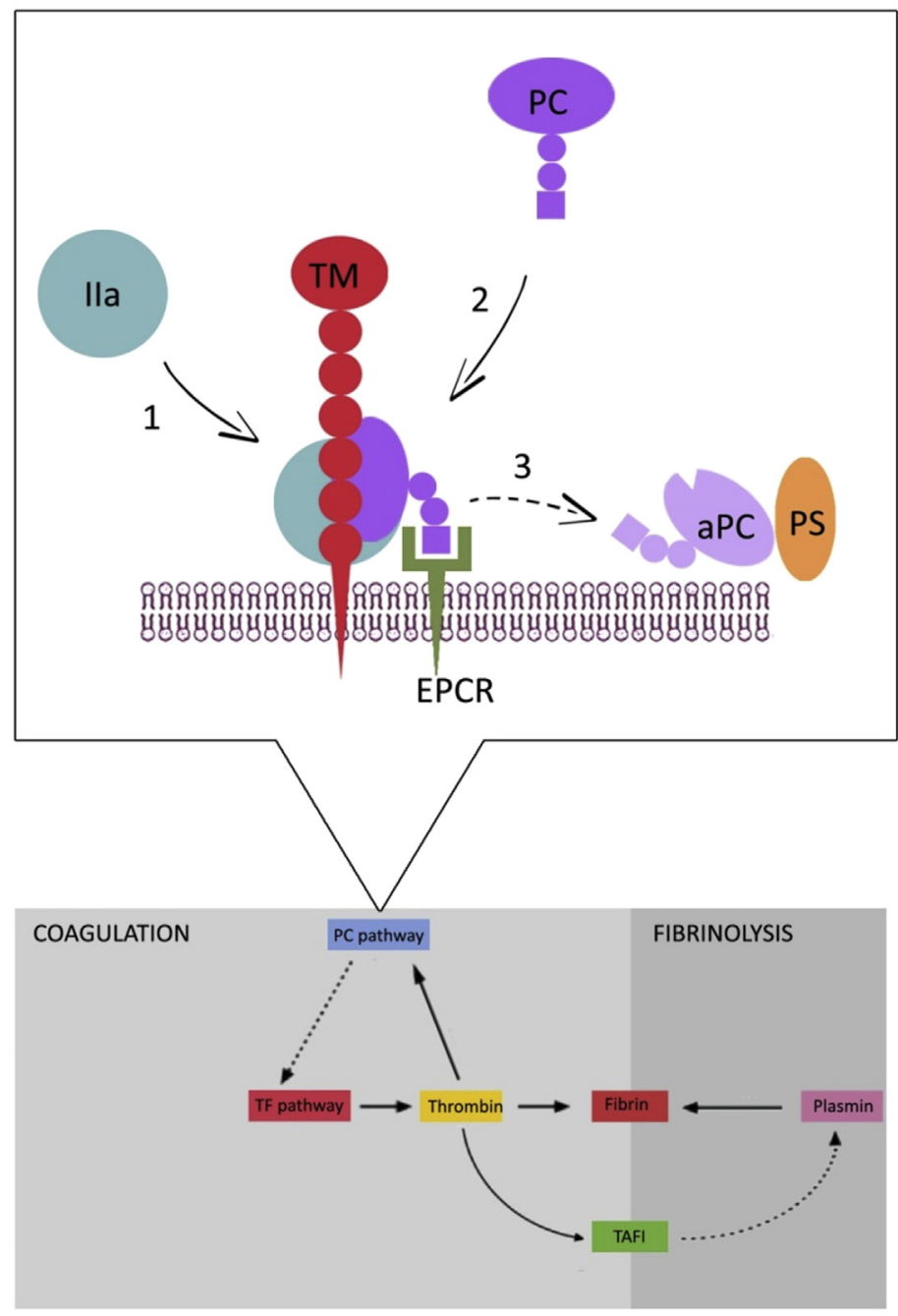

Fig. 3. The protein $C$ pathway. TM binds circulating thrombin (1) and forms a TM-thrombin complex, which activates PC bound to EPCR into aPC (2). APC then dissociates from the EPCR, binds to PS, and forms a complex that inactivates factor Va and factor VIIla (3). aPC, activated protein $C$; $E P C R$, endothelial cell protein $C$ receptor; Ila, thrombin; $P S$, protein $S$; TM, thrombomodulin. (From Boeddha NP, Emonts M, Cnossen MH, et al. Gene Variations in the Protein C and Fibrinolytic Pathway: Relevance for Severity and Outcome in Pediatric Sepsis. Semin Thromb Hemost. 2017;43(1):36-47; with permission.)

killing is the release of neutrophil extracellular traps (NETs). NETs are extracellular DNA matrix, containing granule proteins and histones to degrade virulence factors and to kill bacteria. ${ }^{35}$

Although NETs are primarily considered as a protective mechanism because of the toxicity of antimicrobial components of the NETs, NETs may contribute to disease severity by causing cell damage via cytotoxic effects of NET-bound histones and by promoting coagulation. ${ }^{36-38}$ During systemic inflammation in sepsis, NETs or their components may damage tissue and endothelia, which then initiates the coagulation cascade. 
In addition, NETs stimulate platelet adhesion, which may partly account for the thrombocytopenia commonly observed in sepsis. ${ }^{39} \mathrm{NET}$ s also promote various procoagulant and antifibrinolytic processes, ${ }^{38}$ such as fibrin clot formation, factor XII activation, and via histones, interaction with thrombomodulin-dependent PC activation leading to increased thrombin generation. This process named "immunothrombosis" is an important link between inflammation and coagulation. ${ }^{40}$ Ideally, a balance in NETs is needed to prevent excess thrombin generation, while preserving adaptive hemostasis.

The currently available literature on NETs or components of NETs in sepsis mostly originates from adult studies or animal studies. Several adult studies demonstrated increased (derivatives of) NETs during infectious conditions: increased neutrophil elastase-DNA in patients with pneumonia and nonpulmonary sepsis compared with critically ill control subjects, ${ }^{41}$ increased plasma histone and cell free (cf)-DNA levels in 17 patients with sepsis compared with critically ill patients without sepsis, ${ }^{42}$ elevated cf-DNA and myeloperoxidase DNA in patients with influenza A viral infection, ${ }^{43}$ and higher serum cf-DNA/NETs levels in 31 patients with sepsis compared with healthy control subjects. ${ }^{36}$ In a study including 60 children with meningococcal sepsis, NET levels were higher in the acute phase of disease, that is, at admission to pediatric intensive care unit and at 24 hours after admission, compared with 1 month after admission. ${ }^{44}$ Most of these studies reported an association between NET levels and illness severity or mortality. ${ }^{36,41,43}$ Thus, in the early phase of infection, NETs are increased and seem to be associated with illness severity.

\section{SUMMARY}

This article reviews the inflammatory and hemostatic response in sepsis, illustrating a complex interplay among host, pathogen, and environmental factors. Meningococci hold unique properties to promote adhesion, colonization, and invasion into the bloodstream. The inflammatory host response, including proinflammatory and antiinflammatory responses in innate and adaptive immunity, skew toward a proinflammatory state. This leads to endothelial dysfunction and activation of hemostatic response. Within the wide range of illness severity, deposition of fibrin throughout the microcirculation could result in multiple organ dysfunction, the need for skin grafts and amputation, and eventually death.

\section{DISCLOSURE}

The authors declare no conflicts of interest.

\section{REFERENCES}

1. Martinon-Torres F, Salas A, Rivero-Calle I, et al. Life-threatening infections in children in Europe (the EUCLIDS Project): a prospective cohort study. Lancet Child Adolesc Health 2018;2(6):404-14.

2. Boeddha NP, Schlapbach LJ, Driessen GJ, et al. Mortality and morbidity in community-acquired sepsis in European pediatric intensive care units: a prospective cohort study from the European Childhood Life-threatening Infectious Disease Study (EUCLIDS). Crit Care 2018;22(1):143.

3. Wright V, Hibberd M, Levin M. Genetic polymorphisms in host response to meningococcal infection: the role of susceptibility and severity genes. Vaccine 2009; 27(Suppl 2):B90-102.

4. Emonts M, Hazelzet JA, de Groot R, et al. Host genetic determinants of Neisseria meningitidis infections. Lancet Infect Dis 2003;3(9):565-77. 
5. Loh E, Kugelberg E, Tracy A, et al. Temperature triggers immune evasion by Neisseria meningitidis. Nature 2013;502(7470):237-40.

6. Boeddha NP, Driessen GJ, Cnossen $\mathrm{MH}$, et al. Circadian variation of plasminogen-activator-inhibitor-1 levels in children with meningococcal sepsis. PLoS One 2016;11(11):e0167004.

7. Zeerleder S, Hack CE, Wuillemin WA. Disseminated intravascular coagulation in sepsis. Chest 2005;128(4):2864-75.

8. Dwilow R, Fanella S. Invasive meningococcal disease in the 21st century-an update for the clinician. Curr Neurol Neurosci Rep 2015;15(3):2.

9. Nadel S, Ninis N. Invasive meningococcal disease in the vaccine era. Front Pediatr 2018;6:321.

10. Hotchkiss RS, Monneret G, Payen D. Sepsis-induced immunosuppression: from cellular dysfunctions to immunotherapy. Nat Rev Immunol 2013;13(12):862-74.

11. Netea MG, Balkwill F, Chonchol M, et al. A guiding map for inflammation. Nat Immunol 2017;18(8):826-31.

12. van der Poll T, van de Veerdonk FL, Scicluna BP, et al. The immunopathology of sepsis and potential therapeutic targets. Nat Rev Immunol 2017;17(7):407-20.

13. Muszynski JA, Nofziger R, Moore-Clingenpeel M, et al. Early immune function and duration of organ dysfunction in critically III children with sepsis. Am J Respir Crit Care Med 2018;198(3):361-9.

14. Boeddha NP, Kerklaan D, Dunbar A, et al. HLA-DR expression on monocyte subsets in critically III children. Pediatr Infect Dis J 2018;37(10):1034-40.

15. Venet F, Lukaszewicz AC, Payen D, et al. Monitoring the immune response in sepsis: a rational approach to administration of immunoadjuvant therapies. Curr Opin Immunol 2013;25(4):477-83.

16. Stephens DS, Hoffman LH, McGee ZA. Interaction of Neisseria meningitidis with human nasopharyngeal mucosa: attachment and entry into columnar epithelial cells. J Infect Dis 1983;148(3):369-76.

17. Nassif X, So M. Interaction of pathogenic neisseriae with nonphagocytic cells. Clin Microbiol Rev 1995;8(3):376-88.

18. Schneider MC, Prosser BE, Caesar JJ, et al. Neisseria meningitidis recruits factor H using protein mimicry of host carbohydrates. Nature 2009;458(7240):890-3.

19. Davila S, Wright VJ, Khor CC, et al. Genome-wide association study identifies variants in the $\mathrm{CFH}$ region associated with host susceptibility to meningococcal disease. Nat Genet 2010;42(9):772-6.

20. van Deuren M, Brandtzaeg P, van der Meer JW. Update on meningococcal disease with emphasis on pathogenesis and clinical management. Clin Microbiol Rev 2000;13(1):144-66, table of contents.

21. Iba T, Levy JH. Inflammation and thrombosis: roles of neutrophils, platelets and endothelial cells and their interactions in thrombus formation during sepsis. J Thromb Haemost 2018;16(2):231-41.

22. Gando S, Levi M, Toh CH. Disseminated intravascular coagulation. Nat Rev Dis Primers 2016;2:16037.

23. Levi M, van der Poll T. Coagulation and sepsis. Thromb Res 2017;149:38-44.

24. Mairey E, Genovesio A, Donnadieu E, et al. Cerebral microcirculation shear stress levels determine Neisseria meningitidis attachment sites along the blood-brain barrier. J Exp Med 2006;203(8):1939-50.

25. Nourshargh S, Hordijk PL, Sixt M. Breaching multiple barriers: leukocyte motility through venular walls and the interstitium. Nat Rev Mol Cell Biol 2010;11(5): 366-78. 
26. Coureuil M, Join-Lambert $\mathrm{O}$, Lecuyer $\mathrm{H}$, et al. Pathogenesis of meningococcemia. Cold Spring Harb Perspect Med 2013;3(6) [pii:a012393].

27. Pron B, Taha MK, Rambaud C, et al. Interaction of Neisseria meningitidis with the components of the blood-brain barrier correlates with an increased expression of PilC. J Infect Dis 1997;176(5):1285-92.

28. Esmon CT. The protein C pathway. Chest 2003;124(3 Suppl):26S-32S.

29. Macias WL, Nelson DR. Severe protein C deficiency predicts early death in severe sepsis. Crit Care Med 2004;32(5 Suppl):S223-8.

30. Hermans PW, Hibberd ML, Booy R, et al. 4G/5G promoter polymorphism in the plasminogen-activator-inhibitor-1 gene and outcome of meningococcal disease. Meningococcal Research Group. Lancet 1999;354(9178):556-60.

31. Li L, Nie W, Zhou H, et al. Association between plasminogen activator inhibitor-1 -675 4G/5G polymorphism and sepsis: a meta-analysis. PLoS One 2013;8(1): e54883.

32. Wong HR. Genetics and genomics in pediatric septic shock. Crit Care Med 2012; 40(5):1618-26.

33. Rautanen A, Mills TC, Gordon AC, et al. Genome-wide association study of survival from sepsis due to pneumonia: an observational cohort study. Lancet Respir Med 2015;3(1):53-60.

34. Kovach MA, Standiford TJ. The function of neutrophils in sepsis. Curr Opin Infect Dis 2012;25(3):321-7.

35. Brinkmann V, Reichard U, Goosmann C, et al. Neutrophil extracellular traps kill bacteria. Science 2004;303(5663):1532-5.

36. Czaikoski PG, Mota JM, Nascimento DC, et al. Neutrophil extracellular traps induce organ damage during experimental and clinical sepsis. PLoS One 2016;11(2):e0148142.

37. McDonald B, Davis RP, Kim SJ, et al. Platelets and neutrophil extracellular traps collaborate to promote intravascular coagulation during sepsis in mice. Blood 2017;129(10):1357-67.

38. Papayannopoulos V. Neutrophil extracellular traps in immunity and disease. Nat Rev Immunol 2018;18(2):134-47.

39. Fuchs TA, Brill A, Duerschmied D, et al. Extracellular DNA traps promote thrombosis. Proc Natl Acad Sci U S A 2010;107(36):15880-5.

40. Kimball AS, Obi AT, Diaz JA, et al. The emerging ROLE of NETs in venous thrombosis and immunothrombosis. Front Immunol 2016;7:236.

41. Lefrancais E, Mallavia B, Zhuo H, et al. Maladaptive role of neutrophil extracellular traps in pathogen-induced lung injury. JCl Insight 2018;3(3) [pii:98178].

42. Hashiba M, Huq A, Tomino A, et al. Neutrophil extracellular traps in patients with sepsis. J Surg Res 2015;194(1):248-54.

43. Zhu L, Liu L, Zhang Y, et al. High level of neutrophil extracellular traps correlates with poor prognosis of severe influenza A infection. J Infect Dis 2018;217(3): 428-37.

44. Hoppenbrouwers T, Boeddha NP, Ekinci E, et al. Neutrophil extracellular traps in children with meningococcal sepsis. Pediatr Crit Care Med 2018;19(6):e286-91. 